\title{
EVALUACIÓN DE EFICIENCIA DEL SISTEMA DOMICILIARIO PARA ABASTECIMIENTO DE AGUA SEGURA, CORREGIMIENTO SAN FERNANDO, NARIÑO
}

\author{
EFFICIENCY EVALUATION OF THE DOMICILIARY SYSTEM \\ FOR SAFE WATER SUPPLY, SAN FERNANDO DISTRICT
}

\author{
Álvaro Javier Ceballos Freire \\ Ingeniero agroforestal, Universidad de Nariño \\ aceb1980@udenar.edu.co
}

\begin{abstract}
RESUMEN
Contextualización: las zonas rurales colombianas presentan un déficit en cobertura del servicio de abastecimiento de agua segura o en la eficiencia de los sistemas de tratamiento implementados, en términos de remoción de agentes patógenos.
\end{abstract}

Vacío de conocimiento: las aguas que se usan para el consumo no se tratan adecuadamente, sumado a la deficiente cobertura de abastecimiento de la misma, y a pesar de que la población realiza por cuenta propia el tratamiento del agua, no se garantiza que esté en óptimas condiciones para su consumo.

Propósito del estudio: el objetivo de esta investigación fue determinar la eficiencia de remoción de un sistema domiciliario no convencional para el abastecimiento de agua segura en el corregimiento de San Fernando Municipio de Pasto, Colombia.
Metodología: se desarrollaron tres fases: la primera, estableció el diagnóstico de la zona de estudio, el cual contempló un mapa de riesgo, caracterización de la fuente de abastecimiento, caracterización físico-espacial, socioeconómica y cultural, selección de muestra de la población y formulación de indicadores; la segunda, definió la tecnología por implementar a través del análisis multicriterio y el proceso de análisis jerárquico con seis expertos, donde también se diseñó e implementó la estrategia de transferencia participativa para la higiene y el saneamiento, complementada con los procesos de enseñanza y aprendizaje; y la tercera, determinó la eficiencia del sistema de abastecimiento de agua segura implementado.

Resultados y conclusiones: por medio de tres seguimientos de toma de muestras, y la comparación con las muestras de agua cruda obtenidas en la caracterización, se determinó 
que el sistema de tratamiento es $99 \%$ eficiente en la remoción de turbidez, coliformes totales y coliformes fecales, lo que garantiza el consumo de agua segura en la población. Por otro lado, es importante considerar que el éxito de las tecnologías no convencionales está ligado a la transferencia social que se aplique a lo largo de su implementación; la apropiación que la comunidad le dé al sistema es vital para su sostenibilidad en el tiempo y el seguimiento que se realice al proceso por parte de los líderes sociales de la zona.

Palabras clave: filtro; patógeno; eficiencia; agua potable.

\section{ABSTRACT}

Contextualization: Colombian rural areas have a deficit in the coverage of safe water supply service. In addition, the treatment systems already implemented are not efficient enough in the removal of pathogens.

Knowledge gap: The waters used for consumption are not treated properly, added to the poor coverage of its supply, and despite the fact that the population carries out the water treatment on its own, it is not guaranteed that it is in optimal conditions for its consumption.

Purpose: The objective of this investigation was to determine the removal efficiency of an unconventional household system for the supply of safe water in the San Fernando District - Municipality of Pasto, Colombia.
Methodology: Three phases were developed: the first, was the diagnosis of the study zone, which included a risk map, characterization of the supply source, physical-spatial, socioeconomic and cultural characterization, selection of the population under study and formulation of indicators; the second, defined the technology to be implemented through the multicriteria analysis and hierarchical analysis process with 6 experts, where the participatory transfer strategy for hygiene and sanitation was also designed and implemented, complemented with the teaching-learning strategy; and the third, determined the efficiency of the safe water supply system implemented.

Results and conclusions: By mean of three monitoring samples and the comparison with the raw water samples obtained in the characterization, it was determined that the treatment system was $99 \%$ efficient in the turbidity removal, total coliforms and fecal coliforms, which guarantees the consumption of safe water in this population. On the other hand, it is important to consider that the success of unconventional technologies is linked to the social transfer that is applied throughout their implementation; the appropriation that the community gives to the system is vital for its sustainability over time and for the followup process developed by the social leaders of the area.

Keywords: filter; pathogen; efficiency; Water for consumption. 


\section{RESUMEN GRÁFICO}

Procesos investigativos en la transferencia e implementación de un sistema domiciliario no convencional para abastecimiento de agua segura.

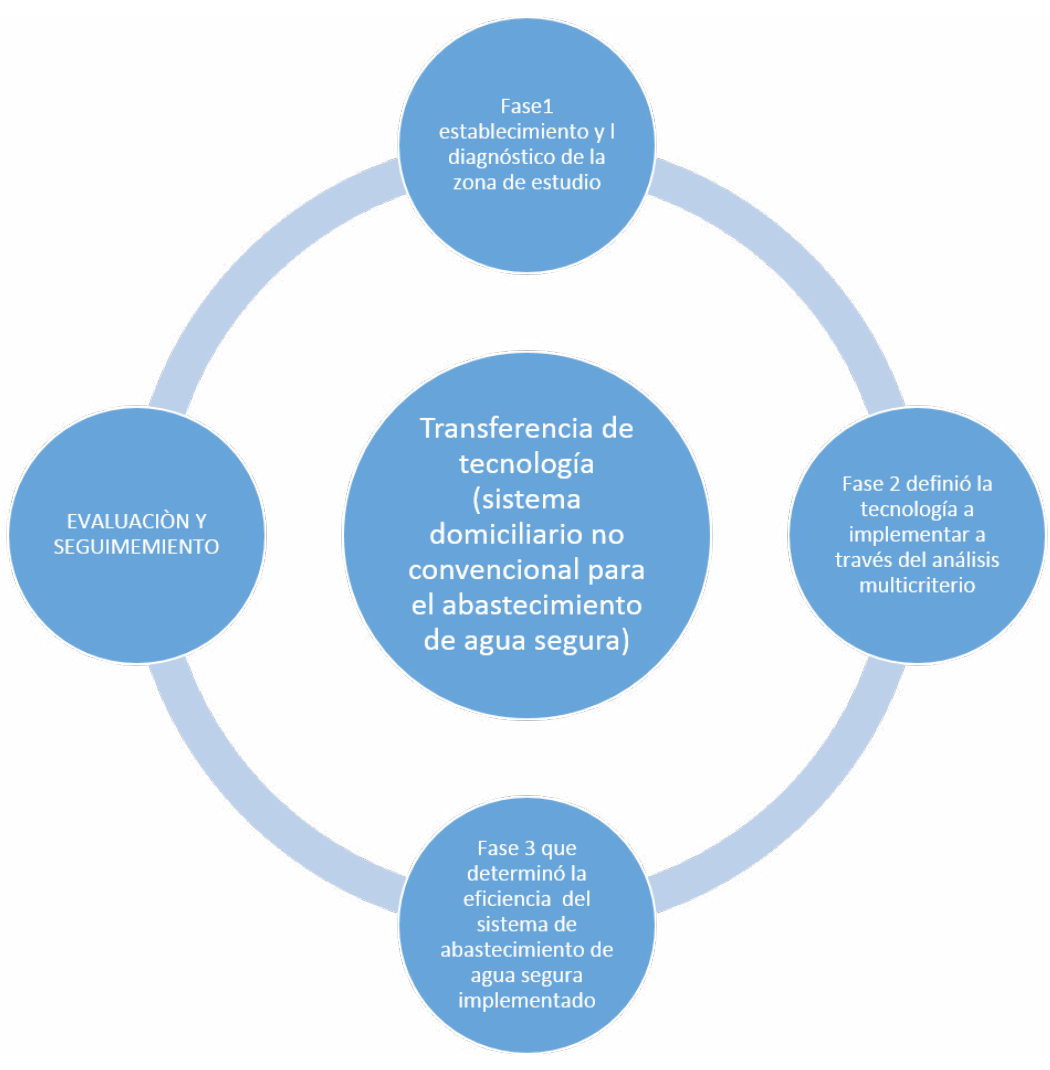

\section{INTRODUCCIÓN}

El recurso hídrico es uno de los elementos esenciales en el sustento de la vida y es fundamental en el desarrollo del ser humano, puesto que requiere diferentes cantidades y calidades específicas según lo demande cada una de las actividades de vital importancia, como lo son el consumo y las actividades productivas, para garantizar así su sostenimiento en el tiempo (Pontificio Consejo de Justicia y Paz, 2012). También afecta los patrones de vida y cultura regionales. Por esto, se le reconoce como un agente preponderante en el desarrollo de las comunidades (FUSDA, 2008). El agua puede resultar alterada fácilmente a causa de procesos antrópicos y naturales, lo cual, en consecuencia, puede repercutir negativamente en la salud humana (Fernández, 2012).

La calidad del agua vendría determinada por la erosión del sustrato mineral, los procesos atmosféricos de evapotranspiración y sedimentación de lodos y sales, la lixiviación natural de la materia orgánica y los nutrientes del suelo por los factores hidrológicos, y los procesos biológicos en el medio acuático que pueden alterar la composición física y química del agua (ONU, 2014). En cuanto a la acción antrópica, la calidad del agua estaría condicionada por varias fuentes, debido a procesos de eutrofización provocados por escorrentías agrícolas, aguas residuales domésticas y efluentes industriales, entre otros (Espinosa, 2014). 
El acceso al agua potable es uno de los derechos humanos básicos y un componente fundamental de las políticas eficaces de protección de la salud en las comunidades (NUDH, 2011). Según la OMS (2004), el agua no debe presentar ningún tipo de alteración ni riesgo que pueda causar irritación química, intoxicación o infección microbiológica que sea perjudicial a la salud humana.

En el departamento de Nariño (Colombia), la cobertura de acueducto urbano es del $89 \%$ y la de acueducto rural del 64\%. La cobertura de agua potable para el departamento fue del $85 \%$ en la zona urbana y del $2,6 \%$ en el área rural, es decir, el $97.4 \%$ restante se abastece de agua que no está catalogada como apta para el consumo humano (Instituto Departamental de Salud de Nariño, 2016).

El corregimiento de San Fernando, localizado en el departamento de Nariño, es un claro ejemplo de la problemática anteriormente mencionada, puesto que no cuenta con una cobertura completa de abastecimiento de agua potable $y$, a pesar de que la población realiza por cuenta propia el tratamiento del agua utilizando únicamente el proceso de cloración, no se garantiza que esté en óptimas condiciones para su consumo (JAL, 2017). Según la Subsecretaría de Planeación y Calidad de Pasto (2018), la población del corregimiento de San Fernando en el año 2017 reportó 148 casos de gastritis, 157 casos de amebiasis y parasitosis, 38 casos de gastroenteritis, 12 casos de estreñimiento, 3 casos de cálculos biliares y 1 caso de colitis.

Las intervenciones de tratamiento doméstico del agua (TDA), pueden contribuir en gran medida a la protección de la salud pública en situaciones en las que el agua de consumo de diversas fuentes, incluso el agua entubada u otras fuentes mejoradas, no recibe un tratamiento adecuado o se contamina durante su distribución o almacenamiento (OMS, 2012). Por todo lo anterior, la implementación de sistemas de tratamiento de agua de tipo no convencional se convierte en una alternativa viable para la depuración de agentes patógenos, garantizando así las condiciones óptimas para el consumo o preparación de alimentos.

Por esto, esta investigación tuvo como objetivo evaluar un sistema domiciliario de tipo no convencional para el mejoramiento de la calidad de agua de consumo humano en población vulnerable en el municipio de Pasto, corregimiento de San Fernando. Se realizó un diagnóstico integral enfocado al abastecimiento y consumo de agua y posterior a ello, un seguimiento del sistema domiciliario de tipo no convencional de agua para consumo. Se determinó la eficiencia de la tecnología y la transferencia social a la población objeto de estudio, a partir del diagnóstico de la comunidad, definición de la tecnología y la determinación del porcentaje de eficiencia en la remoción de patógenos en el agua de consumo.

\section{MATERIALES Y MÉTODOS}

Esta investigación se realizó en la vereda de Alto San Fernando en el corregimiento de San Fernando, que tiene una población de 250 habitantes equivalente a 90 familias, localizada en el municipio de Pasto (Nariño, Colombia) a 4 kilómetros de la ciudad de San Juan de Pasto con una altura de 2800 m.s.n.m., su posición geográfica es de $1^{\circ} 13^{\prime} 14.78^{\prime \prime}$ latitud norte y $77^{\circ} 13^{\prime} 35^{\prime \prime \prime}$ longitud oeste, presenta un clima frío con temperaturas que se establecen entre $10^{\circ} \mathrm{C}$ y $14^{\circ} \mathrm{C}(\mathrm{JAL}, 2017)$.

Para dar cumplimiento al objetivo de esta investigación se desarrollaron tres fases, la primera de ellas el diagnóstico; la segunda, la 
definición de la tecnología; y la tercera, la determinación de la eficiencia de la tecnología. Dentro del análisis y elaboración de las figuras se usó el software Excel de Microsoft.

El diagnóstico integral propuesto en la investigación incluye los aspectos sociales, culturales, económicos, ambientales en la evaluación y el análisis situacional de cada familia involucrada en la investigación. Se aplicó una encuesta semiestructura con múltiples respuestas orientadas a la selección de una de ellas, la cual se abordó desde diferentes contextos, teniendo en cuenta ítems de vulnerabilidad, ingresos, condición de la familia, presencia de adultos mayores y niños (núcleo familiar).

Se utilizó la técnica jerárquica ponderada, en la cual se incluyeron criterios cualitativos y cuantitativos, para lograr una única decisión en cuanto a la tecnología a incorporar en la investigación, incluyendo aspectos profesionales (expertos), económicos, técnicos y ambientales que llevaron a un análisis matemático para dicha decisión.

\section{ANÁLISIS ESTADÍSTICOS DE CADA UNA DE LAS FASES}

\section{Fase diagnóstica}

Caracterización físico espacial económica y cultural de la comunidad: la primera fase de elaboración de este diagnóstico integral se basó en el manual de introducción a la gestión ambiental municipal propuesto por el CEPPIA (2011), establece la calidad de la fuente de abastecimiento y su influencia en la población de la zona.

Se utilizó un enfoque metodológico mixto que combina la revisión de información secundaria con la obtención de información primaria a través de la aplicación de encuestas y entrevistas semiestructuradas a actores clave. La información secundaria se recopiló con base en la suministrada por El Instituto Departamental de Salud de Nariño (2016) a través de sus canales oficiales y el último censo sanitario (JAL, 2017).

Se diseñó una encuesta semiestructurada con 42 preguntas de única respuesta y selección múltiple, orientada a generar información sobre aspectos sociales, características de la familia, condiciones de vivienda, agua para consumo humano, saneamiento básico y cultura (ver Archivo suplementario 1). Para esto se consideraron aspectos basados en las metodologías propuestas y ejecutadas por Patiño et al. (2015) y León-Agatón et al. (2015).

Selección de muestra de estudio: la muestra objeto de estudio, se calculó mediante la fórmula propuesta por Lagares \& Puerto (2001) (ver Archivo Suplementario 1), teniendo en cuenta, un grado de confianza del $90 \%$ (Z), una probabilidad de ocurrencia del $90 \%(p)$ y un error estándar estimado del $10 \%$ (e), en este sentido se obtuvo un total de 90 familias por intervenir, la ponderación de criterios de selección de beneficiarios se realizó a través de un muestreo no paramétrico (Trejos, 2007) en tres etapas.

En la primera fase se identificaron las características de la población en los 90 hogares seleccionados a través de las encuestas programadas; en la segunda etapa, mediante el software Excel 2013, se estableció un mecanismo técnico para el registro de beneficiarios potenciales, codificando las variables a través de categorías exclusivas e independientes a cada pregunta de la encuesta. Por último, se calificó con un valor de 1 a las familias que presentaban los siguientes criterios: 
población más vulnerable (población infantil y adultos mayores), ingresos económicos en el hogar menores a un S.M.L.V, si el hogar no tiene acceso a un sistema de salud, estrato social uno, si no cuenta con acceso de agua segura y condiciones de la vivienda (piso en barro - paredes en tapia); los hogares con mayor puntaje fueron los seleccionados. Por otro lado, una vez se conoció la vulnerabilidad y las condiciones de vida de las familias, se definió los criterios económicos, técnicos, sociales y ambientales que apoyaron la selección de la tecnología.

Con base en la información recopilada, se realizó el mapa de riesgo de calidad de agua para consumo humano (ver Archivo suplementario 1), a través de la metodología propuesta por Carvajal (2012) y la Resolución 4716 de 2010 expedida por el Ministerio de Ambiente, Vivienda y Desarrollo Territorial (MINAMBIENTE, 2010).

Para ello se usó el software ArcGis versión 10.1 a escala $1: 25.000$ en donde, se identificaron los parámetros fisicoquímicos y microbiológicos por analizar, de acuerdo con la Resolución 2115 de 2007 y el Decreto 1575 de 2007, expedido por el Ministerio de la Protección Social y Ministerio de Ambiente, Vivienda y Desarrollo Territorial (MAVDT, 2007) y el Ministerio de la Protección Social (2007), en las dos bocatomas de abastecimiento del corregimiento de San Fernando.

De igual forma, se realizó un muestreo en el punto de abastecimiento directo en una vivienda al azar, posteriormente fueron llevados a un laboratorio certificado por la NTCISO 5667; finalmente se estableció la línea base de las condiciones iniciales en cuanto a la calidad del agua.

\section{Caracterización físico-espacial, socioeconómica y cultural}

\section{Fase definición de la tecnología}

En la segunda fase se definió la tecnología a través del modelo multicriterio propuesto por Mazorra \& González (2012); para el desarrollo de este modelo en primera instancia se definieron seis expertos en temas de abastecimiento seguro de agua, saneamiento $y$ de entorno saludable. Paralelamente, se efectuó una revisión bibliográfica que identificó diferentes sistemas domiciliarios de tipo no convencional para consumo de agua segura y se realizó un catálogo de alternativas que puedan ser efectivas en el tratamiento de agua potable con sus respectivas características.

Posteriormente, se hizo una consulta con estos expertos para preseleccionar cuatro estrategias viables por medio de la técnica de análisis jerárquico ponderado propuesta por Moreno (2012) (ver Archivo Suplementario 1). Para esta jerarquización, se asignó a cada una de las estrategias un puntaje del uno al diez, donde el puntaje de diez indicaba la mayor importancia y el puntaje uno, la menor importancia; no se repitió ningún puntaje. Una vez se obtuvieron las cuatro estrategias, los expertos calificaron los pesos de criterios formulados en la fase de diagnóstico:

- Técnico: qué tan eficiente es la remoción de agentes patógenos.

- Ambiental: la no generación de residuos que puedan afectar el medio ambiente.

- Económico: cuáles son los costos de adquisición, operación, mantenimiento y si representa ingresos o egresos.

- Social: Si minimiza la vulnerabilidad ante posibles enfermedades. 
Para efectos de la calificación, se tuvo en cuenta la intensidad de importancia (Tabla 1) y el razonamiento de opuestos que dicta: si la fila es absolutamente más importante que la columna y tiene una potencia de 9 , entonces la columna tiene que ser absolutamente menos importante que la fila y está valorado en $1 / 9$ (Mazorra \& González, 2012).

Tabla 1. Intensidad de criterios.

\begin{tabular}{|c|c|c|}
\hline Intensidad de importancia & Definición & Explicación \\
\hline 1 & Igual importancia & $\begin{array}{l}\text { Dos factores contribuyen } \\
\text { igualmente en el objetivo }\end{array}$ \\
\hline 3 & Algo más importante & $\begin{array}{l}\text { La experiencia y el juicio favorecen } \\
\text { ligeramente uno sobre el otro }\end{array}$ \\
\hline 5 & Mucho más importante & $\begin{array}{l}\text { La experiencia y el juicio favorecen } \\
\text { fuertemente uno sobre el otro }\end{array}$ \\
\hline 7 & Muchísimo más importante & $\begin{array}{l}\text { La experiencia y el juicio favorecen muy } \\
\text { fuertemente sobre el otro. Su importancia } \\
\text { se demuestra en la practica }\end{array}$ \\
\hline 9 & Absolutamente más importante & $\begin{array}{l}\text { La evidencia a favor de uno sobre el otro es } \\
\text { de la validez más alta posible }\end{array}$ \\
\hline
\end{tabular}

Fuente: Moreno, 2012.

Una vez se ejecutó la ponderación de los criterios, los seis expertos evaluaron los cuatro (4) sistemas seleccionados de acuerdo a cada uno de los criterios técnico, ambiental, económico y social. Igualmente, para efectos de la calificación se tuvo en cuenta la intensidad de criterios (Tabla 1) y el razonamiento de opuestos.

Al obtener la calificación por cada experto, los resultados fueron transcritos en una matriz de datos en el software Excel 2013, los cuales, se normalizaron mediante la función "Normalización", con el fin de ajustar los valores de cada columna (variable) con base en la media y desviación estándar de cada grupo de datos completos. Posteriormente, se obtuvo el vector de prioridad a través del promedio de los valores de la matriz normalizada. Para el valor del vector de prioridad de cada tecnología, se calculó su ponderado y este a su vez se multiplicó por el valor del vector de prioridad de cada criterio, obteniendo así el sistema a seleccionar a través de la generación de un gráfico radial realizado en el mismo software. En este se puede visualizar el aporte de cada sistema sobre los criterios establecidos en la fase diagnostica y su orden de importancia a través de un ranking descendente, en donde el de mayor valor sea el mejor.

\section{Implementación de la estrategia de transferencia social}

En relación con la ejecución de una adecuada transferencia social hacia la comunidad, se establecieron cinco reuniones. En la primera, se realizó un acercamiento con los líderes comunitarios y la comunidad seleccionada, considerando que generar un ambiente social de aceptación por parte de la comunidad aumenta la probabilidad de éxito de apropiación de la estrategia social (Murtagh, 2009). Se realizó entonces la socialización del proyecto, donde se presentó el equipo de trabajo, la justificación del desarrollo 
de la investigación, las actividades que se llevarían a cabo en la zona de estudio, las características de los sistemas de tratamiento doméstico de agua y la importancia de la higiene y saneamiento a través de un sociodrama con actividades lúdicas y se consolidó el compromiso de la comunidad con la participación activa en el proyecto.

En la segunda reunión con la comunidad, se organizó una charla para presentar el sistema no convencional por implementar, sus características, y propiedades fundamentales. Asimismo, se explicó la importancia de implementarlo en cada hogar y se habló sobre los conceptos básicos de uso y mantenimiento de los mismos.

En la tercera, cuarta y quinta reunión con la comunidad, se llevó a cabo el desarrollo de la guía de transferencia participativa para la higiene y el saneamiento, propuesta por la Organización Mundial de la Salud (2012) y fue complementada con los procesos de enseñanza aprendizaje propuesta por Murtagh (2009) con el fin de lograr una adecuada apropiación de la tecnología. Cabe resaltar que también se realizaron seguimientos mensuales para determinar si se ha presentado un adecuado uso y mantenimiento por parte de la comunidad mediante un formato de evaluación técnico propuesto por (Leonel et al., 2016).

Para la tercera y última fase de la determinación de la eficiencia de la tecnología se contempló: la selección, capacitación y el seguimiento en su instalación, uso y manejo (metodología Organización Mundial de la Salud 2012), para lo cual se tomaron muestras de agua del sistema de abastecimiento implementado, y se compararon con las muestras de agua sin tratamiento obtenidas en la caracterización de la calidad del agua, en comparación con la normativa (Resolución 2115/MAVDT, 2007).

\section{RESULTADOS Y DISCUSIÓN}

Fase diagnóstica: la vereda Alto San Fernando limita al nororiente con el corregimiento de La Laguna, al norte con las veredas, La Cadena y El Común, al sur oriente con el corregimiento de Mocondino. La comunidad se abastece con dos bocatomas, ubicadas en de la quebrada Tábano y Chauperrio, captando un caudal de 11 l/s. El acueducto se construyó hace 40 años y desde hace 14 años no se realiza el mantenimiento a la red de tubería que distribuye el agua (JAL, 2017). El acueducto es administrado por una Junta local y se financia a través de recursos de la comunidad.

Para la fase de diagnóstico se realizó el mapa de riesgo de acuerdo a la Resolución 4716 de 2010 (MINAMBIENTE, 2010) (Anexo 1); aguas arriba de las bocatomas no se presenta ningún factor antrópico que pueda alterar la calidad del agua; justo antes de la primera bocatoma hay una caída de agua de aproximadamente 30 metros. Según Sánchez (2016) la caída de agua y el choque con rocas mejora la calidad del agua al aumentar las concentraciones de oxígeno disuelto en los componentes de los caudales.

Por otro lado, el agua que ingresa a la bocatoma ubicada sobre la quebrada Tábano presenta dicho beneficio; en la segunda bocatoma se registró un movimiento masal que arrasó con la capa vegetal que cubría el cuerpo de agua. Los predios aledaños a las bocatomas son propiedad de CORPONARIÑO y hacen parte de una zona de conservación.

Por lo anterior, se estableció que los parámetros por analizar para determinar la calidad del agua, teniendo en cuenta puntaje de riesgo, son: $\mathrm{pH}$ (1.5), color aparente (6), alcalinidad (1), hierro (1.5), sulfatos (1), cloruros (1), cloro residual (1.5), dureza total (1), turbidez (15), coliformes totales (15) y E. Coli (25), según el MAVDT (Resolución 2115 de 2007). 
Calidad de la fuente de abastecimiento: la caracterización fisicoquímica realizada a las dos bocatomas determinó que los parámetros fisicoquímicos están dentro de lo estipulado por la normativa. Sin embargo, los parámetros microbiológicos como los coliformes totales y E. Coli la incumplen. El Índice de Riesgo de la Calidad del Agua (IRCA) para las dos fuentes hídricas es de $48,5 \%$ con una clasificación de riesgo alto para consumo humano, de acuerdo al Artículo 15 de la Resolución 2115 de 2007. La red de distribución a lo largo de su trayecto por toda la vereda presenta alteraciones microbiológicas y estas se agravan a medida que el agua llega al tramo más bajo, donde se presenta además la alteración de los parámetros fisicoquímicos como el color aparente y turbidez, incumpliendo la norma.

Las estructuras de red de distribución de agua pueden realizar un proceso de colmatación al no presentar un adecuado mantenimiento, esto genera una contaminación al contacto con el agua (UNICEF, 2005). La red de distribución de la vereda Alto San Fernando presenta dicha problemática; al no presentarse un mantenimiento de la tubería hace 14 años, el agua tratada se vuelve a contaminar, posiblemente por el proceso de colmatación de la tubería y por medio de conexiones erradas. El índice de riesgo se mantiene en un nivel alto (Tabla 2).

Tabla 2. Calidad de la fuente de abastecimiento.

\begin{tabular}{|c|c|c|c|c|c|c|c|}
\hline \multicolumn{7}{|c|}{ Calidad de la fuente } & \multirow{3}{*}{$\begin{array}{c}\text { Normatividad } \\
\begin{array}{c}\text { Resolución } 2115 \\
\text { de } 2007\end{array}\end{array}$} \\
\hline \multirow{2}{*}{ Parámetro } & \multirow{2}{*}{ Unidades } & \multicolumn{2}{|c|}{ Bocatomas } & \multicolumn{3}{|c|}{ Agua en red } & \\
\hline & & B1 & B2 & C1 & $\mathbf{C 2}$ & C3 & \\
\hline $\mathrm{pH}$ & $\mathrm{pH}$ & 6,9 & 6.8 & 7,3 & 6,6 & 7,3 & Cumplen \\
\hline Color aparente & UPC & 3 & 3,6 & 8 & 4,1 & $* 31$ & Cumplen - *No cumple \\
\hline Turbidez & NTU & 0,26 & 0,21 & 1,39 & 0,34 & $2,66 *$ & Cumplen- $*$ No cumple \\
\hline Alcalinidad & $\mathrm{mg} \mathrm{CaCO}_{3} / \mathrm{l}$ & 62 & 54 & 22 & 56 & 22 & Cumplen \\
\hline Hierro & $\mathrm{mg} \mathrm{Fe} / \mathrm{l}$ & 0,14 & 0,13 & 0,14 & 0,1 & 0,28 & Cumplen \\
\hline Sulfatos & $\mathrm{mg} \mathrm{SO}_{4}{ }^{-2} / \mathrm{l}$ & 1 & 1 & 1 & 1,4 & 1 & Cumplen \\
\hline Cloruros & $\mathrm{mg} \mathrm{Cl} / / \mathrm{l}$ & 9,7 & 8,7 & 1,5 & 6,3 & 1,5 & Cumplen \\
\hline Cloro residual & $\mathrm{mg} / \mathrm{Cl}_{2} / \mathrm{l}$ & 0,03 & 0,02 & 0,01 & 1,1 & 0,02 & Cumplen \\
\hline Dureza total & $\mathrm{ppm} \mathrm{CaCO}$ & 32 & 34 & 24 & 28 & 20 & Cumplen \\
\hline Coliformes totales & $\mathrm{NMP} / 100 \mathrm{ml}$ & 17 & 27 & 3 & 48 & 150 & No cumplen \\
\hline E. coli & $\mathrm{NMP} / 100 \mathrm{ml}$ & 1 & 1 & 3 & 74 & 64 & No cumplen \\
\hline IRCA & $\%$ & 48,2 & 48,2 & 48,2 & 48,2 & 73,5 & \\
\hline $\begin{array}{l}\text { Clasificación } \\
\text { del riesgo }\end{array}$ & Nivel & Alto & Alto & Alto & Alto & Alto & \\
\hline
\end{tabular}


De las encuestas realizadas, se pudo determinar que el $96 \%$ de personas consume agua del sistema de acueducto; entendiendo que la calidad del agua en la red de distribución no es apta para consumo humano, según la caracterización realizada se encuentra una problemática sanitaria. La OMS (2012) establece que el agua no apta para consumo humano y el saneamiento deficiente, están relacionados con la transmisión de enfermedades como cólera, enfermedades diarreicas agudas (EDA), disentería, hepatitis $A$, fiebre tifoidea y poliomielitis.

El deterioro de la salud lleva a que las comunidades retrasen su desarrollo económico, social y cultural, puesto que afecta la normalidad de sus actividades productivas tales como su trabajo, educación y recreación. Además, cuando los infantes adquieren enfermedades, se deteriora su desarrollo físico y emocional (Alleyne \& Cohen, 2003).

Cabe resaltar que el Plan de Desarrollo Municipal (Alcaldía municipal de Pasto, 2016-2019) no estableció proyectos para la construcción de una planta de tratamiento de agua potable en el corregimiento de San Fernando. Por lo anterior, la implementación de tratamientos domésticos de agua se vuelve una alternativa ideal que, de manera inmediata y momentánea, permite a las comunidades tener acceso a agua segura para evitar problemas de salubridad y a la vez mejorar su calidad de vida mientras se les da una solución definitiva (Grisales, 2009).

Caracterización físico-espacial, socioeconómica y cultural: según la clasificación de suelo rural del Plan de Ordenamiento Territorial (POT) de Pasto, "Territorio Con-Sentido (2014-2027)", se pudo determinar que la vereda tiene suelo apto para actividades como la agricultura, el turismo, la ganadería, forestales y la industria. Presenta vías de tipo primaria y secundaria y se ubica a 2 kilómetros de la variante que conecta con el casco urbano de Pasto. Con el fin de conocer los ingresos y las condiciones de habitabilidad de las familias, se determinó que el $85 \%$ de las familias cuenta con vivienda propia. El $70 \%$ de las familias cuenta con 2 a 3 habitaciones. El $67 \%$ de las viviendas están construidas en materiales de cemento y ladrillo, el $23 \%$ en tapia y el $10 \%$ en madera. El $90 \%$ de las viviendas tiene piso en cemento y granito. Por otra parte, el $85 \%$ de las familias de la vereda Alto San Fernando tienen ingresos menores a 1 S.M.L.V (Alcaldía de Pasto, 2014).

Las instituciones que se presentan en la vereda son la Gobernación de Nariño, Alcaldía de Pasto, Instituto Departamental de Salud, CORPONARIÑO, Universidad de Nariño, Universidad Mariana, SENA, hogar comunitario del Instituto Colombiana de Bienestar Familiar (ICBF), Institución Educativa Municipal San Fernando; también cuenta con la Junta de Administradora de acueducto y la Junta de Acción Comunal.

Selección de la muestra de estudio: la muestra significativa para la investigación, según lo propuesto por Lagares \& Puerto (2001), fue de 15 familias, y se seleccionó con base en los requerimientos y vulnerabilidades asociados a la infraestructura y manejo en el consumo de agua en relación con el núcleo familiar e individuos con alto grado de susceptibilidad (edad).

De las 90 familias, una vez ejecutada la metodología de ponderación de criterios para la selección de beneficiarios propuesta por Trejos (2007), nueve familias tuvieron calificaciones de 9 puntos, dos familias con calificaciones de 8,5 puntos y cuatro familias con calificaciones de 8 puntos, siendo estas las 15 familias beneficiarias para ser la muestra objeto de estudio al tener la mayor puntuación. 
Fase determinación de la tecnología

Análisis multicriterio: los expertos dieron un $40,391 \%$ de prioridad al criterio técnico, un $25,579 \%$ al criterio social, un $25,452 \%$ al criterio económico y un $8,578 \%$ al criterio ambiental (Anexo 1).

Los criterios técnico y social establecen el valor más alto, puesto que los expertos consideran que, en el desarrollo de un proceso que busca establecer un sistema adecuado en el abastecimiento de agua segura, lo importante es encontrar una tecnología eficiente para remover agentes patógenos y que sea de fácil apropiación por parte de la comunidad, con el fin de mantener los sistemas en óptimas condiciones y garantizar su sostenibilidad en el tiempo.

Cabe resaltar que, de igual forma, los expertos señalan que el criterio económico no tuvo gran discrepancia respecto al criterio social puesto que el desarrollo de estas investigaciones está muy ceñido a la disponibilidad de recursos. Por otra parte, el criterio ambiental tuvo el puntaje más bajo al considerarse que la generación de residuos no iba a ser a gran escala y que el beneficio que se obtendría sería mucho mayor.

Las alternativas del sistema de tratamiento no convencional analizadas fueron: filtro sawyer; filtro de cerámica tipo vela; filtro lifestraw family; filtro sifón; filtro de bioarena; filtro de cerámica tipo olla; filtro nerox; filtro max power; purificador watly; y purificador de agua genesse de las cuales se preseleccionaron la opción A. filtro sawyer, opción B. filtro lifestraw family, opción $C$. filtro sifón y opción $D$. filtro de bioarena (Tabla 3).

Tabla 3. Preselección de las tecnologías no convencionales para abastecimiento de agua segura.

\begin{tabular}{|c|c|c|c|c|c|c|c|}
\hline \multirow{2}{*}{ Tecnologías } & \multicolumn{6}{|c|}{ Experto } & \multirow{2}{*}{ Total } \\
\hline & 1 & 2 & 3 & 4 & 5 & 6 & \\
\hline Filtro sawyer & 10 & 8 & 10 & 10 & 10 & 10 & 58 \\
\hline Filtro de cerámica tipo vela & 5 & 9 & 1 & 4 & 5 & 7 & 31 \\
\hline Filtro lifestraw family & 9 & 5 & 8 & 9 & 8 & 6 & 45 \\
\hline Filtro sifón & 6 & 6 & 5 & 7 & 7 & 8 & 39 \\
\hline Filtro de bioarena & 8 & 4 & 9 & 8 & 9 & 5 & 43 \\
\hline Filtro de cerámica tipo olla & 1 & 2 & 2 & 5 & 6 & 3 & 19 \\
\hline Filtro nerox & 7 & 3 & 7 & 6 & 2 & 4 & 29 \\
\hline Filtro max power & 2 & 10 & 4 & 1 & 1 & 1 & 19 \\
\hline Purificador watly & 3 & 1 & 3 & 2 & 3 & 9 & 21 \\
\hline Purificador genesse & 4 & 7 & 6 & 3 & 4 & 2 & 26 \\
\hline
\end{tabular}

Fuente: autor

De acuerdo con su injerencia sobre cada criterio, las tecnologías se calificaron así: opción A, correspondiente al filtro sawyer, fue la tecnología con mayor puntuación, con un 43,30\%; seguido de la opción D correspondiente al filtro de bioarena con un 23,87\%; luego la opción C correspondiente al filtro sifón con un 18,60\%; y finalmente la opción B correspondiente al filtro lifestraw family con un $14,87 \%$ (Figura 1 ). 


\section{Selección de Tecnologías}

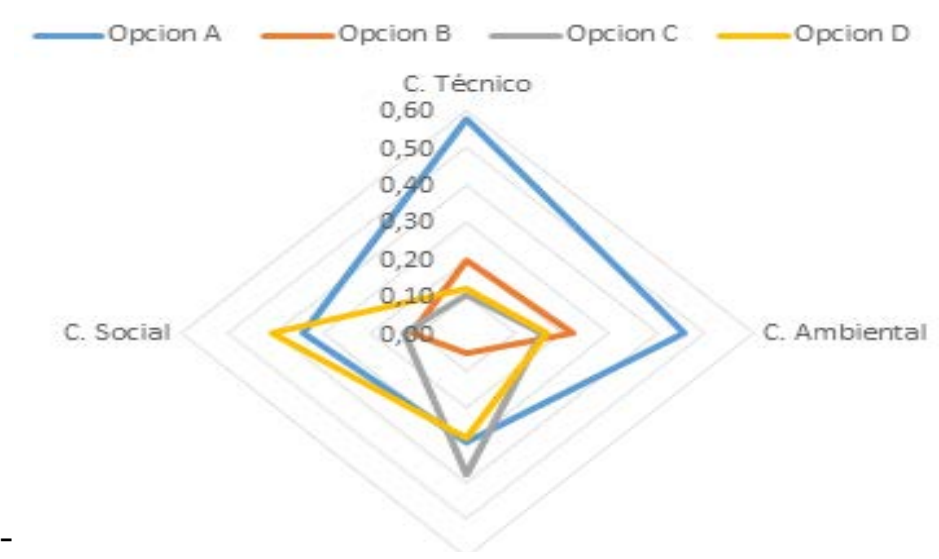

Figura 1. Selección de tecnologías no convencionales para abastecimiento de agua segura: opción A. filtro sawyer, opción B. filtro lifestraw family, opción C. filtro sifón y opción D. filtro de bioarena.

Fuente: autor.

El filtro sawyer fue escogido como la tecnología más adecuada para atender las necesidades que presenta la zona alta del corregimiento de San Fernando. Dicha tecnología prevaleció con un mayor puntaje en los criterios técnico, social y ambiental. Puesto que el filtro presenta mayores beneficios en comparación con las demás alternativas.

Técnicamente el filtro tiene una mayor tasa de filtración con $180 \mathrm{~L} / \mathrm{h}$ respecto a $10 \mathrm{~L} / \mathrm{h}$ del filtro LifeStraw Family, $40 \mathrm{~L} / \mathrm{h}$ del filtro de bioarena y $20 \mathrm{~L} / \mathrm{h}$ del filtro sifón (CAWST, 2011) y ambientalmente no genera residuos al no requerir repuestos. Cabe resaltar que el filtro Sawyer por su forma y diseño tiene una mayor adaptabilidad de acogida por parte de la población, no necesita repuesto a excepción del recipiente de almacenamiento y su mantenimiento es un proceso de retrolavado sencillo (CAWST, 2011).

En el análisis beneficio/costo $(B / C)$, el filtro sawyer posee la mayor relación $B / C$ con un 13,67 , y los valores mayores a 1 representan más beneficios que egresos (Bejarano \& Cisneros, 2013), de igual manera, su costo anual equivalente (CAE) es el menor, con COP 331.383, lo que representa un menor costo en su adquisición, operación y mantenimiento a lo largo de su vida útil. Se tuvieron en cuenta los valores de costo, duración y mantenimiento que especifica el proveedor.

\section{Transferencia social de la tecnología} por parte de la comunidad: para generar una transferencia social de la tecnología en el contexto de la vereda Alto San Fernando, la implementación de la transferencia participativa para la higiene y el saneamiento PHAST (Tabla 4) (ver Archivo suplementario 1) integró la identificación de aspectos ambientales, sociales, institucionales e instrumentos de comunicación. Según Narváez y Martínez (2017), la metodología PHAST brinda una flexibilidad en la ejecución de las actividades y en la selección de herramientas por emplear, las cuales deben adaptarse al contexto socioambiental en el cual se aplica la metodología (anexo 1). 
Tabla 4. Pasos para la transferencia participativa para la higiene y el saneamiento.

Paso Actividad

Herramienta

Resultado

\begin{tabular}{|c|c|c|c|}
\hline $\begin{array}{l}\text { 1. Identificación } \\
\text { del problema }\end{array}$ & $\begin{array}{l}\text { 1. Historias de la comunidad } \\
\text { sobre problemas de salud } \\
\text { en la comunidad }\end{array}$ & $\begin{array}{l}\text { 1. Narración } \\
\text { de anécdotas }\end{array}$ & $\begin{array}{l}100 \% \text { de la comunidad } \\
\text { comentó sus experiencias } \\
\text { respecto a la } \\
\text { problemática sanitaria } \\
\text { en su región. }\end{array}$ \\
\hline $\begin{array}{l}\text { 2. Análisis } \\
\text { del problema }\end{array}$ & $\begin{array}{l}\text { 1. Buenos y malos } \\
\text { hábitos de higiene } \\
\text { 2. Prácticas de la comunidad } \\
\text { 3. Cómo se propagan las } \\
\text { enfermedades }\end{array}$ & $\begin{array}{l}\text { 1. Calificación } \\
\text { en tres grados } \\
\text { 2. Votación con tarjetas } \\
\text { 3. Rutas de transmisión }\end{array}$ & $\begin{array}{l}100 \% \text { de la comunidad } \\
\text { reconoció los malos } \\
\text { hábitos de higiene y } \\
\text { cómo se propagan las } \\
\text { enfermedades }\end{array}$ \\
\hline $\begin{array}{l}\text { 3. Planificación } \\
\text { de soluciones }\end{array}$ & $\begin{array}{l}\text { 1. Cómo detener la propagación } \\
\text { de enfermedades } \\
\text { 2. Selección de las barreras } \\
\text { 3. Tareas de hombres y mujeres } \\
\text { en la comunidad }\end{array}$ & $\begin{array}{l}\text { 1. Bloqueo de las rutas } \\
\text { 2. Gráfico de las barreras } \\
\text { 3. Análisis de género }\end{array}$ & $\begin{array}{l}\text { El 93\% de la comunidad } \\
\text { comprendió como evitar } \\
\text { la propagación } \\
\text { de enfermedades. }\end{array}$ \\
\hline $\begin{array}{l}\text { 4. Selección } \\
\text { de opciones }\end{array}$ & $\begin{array}{l}\text { 1. Elección de las mejoras } \\
\text { de saneamiento } \\
\text { 2. Elección de mejores } \\
\text { hábitos de higiene } \\
\text { 3. tiempo dedicado a preguntas }\end{array}$ & $\begin{array}{l}\text { 1. Opciones de } \\
\text { saneamiento } 2 \text {. } \\
\text { Calificación en } \\
\text { tres grados } \\
\text { 3. Lluvia de preguntas }\end{array}$ & $\begin{array}{l}\text { El } 100 \% \text { de la comunidad } \\
\text { comprendió los hábitos } \\
\text { saludables que se deben } \\
\text { implementar en los } \\
\text { hogares }\end{array}$ \\
\hline $\begin{array}{l}\text { 5. Planificación } \\
\text { de nuevas } \\
\text { instalaciones } \\
\text { y cambios de } \\
\text { comportamiento }\end{array}$ & 1. Planificación para el cambio & $\begin{array}{l}\text { 1. Socialización } \\
\text { de estrategias }\end{array}$ & $\begin{array}{l}\text { El } 93 \% \text { de la comunidad } \\
\text { planificó las estrategias } \\
\text { para prevenir } \\
\text { enfermedades. }\end{array}$ \\
\hline $\begin{array}{l}\text { 6. Planificación } \\
\text { del monitoreo } \\
\text { y evaluación }\end{array}$ & $\begin{array}{l}\text { 1. Preparación para evaluar } \\
\text { el progreso de la comunidad }\end{array}$ & 1. Formato de monitoreo & $\begin{array}{l}100 \% \text { de la comunidad } \\
\text { aprobó el formato de } \\
\text { monitoreo }\end{array}$ \\
\hline $\begin{array}{l}\text { 7. Evaluación } \\
\text { de la planificación }\end{array}$ & 1. Evaluación del progreso & $\begin{array}{l}\text { 1. Ejecución del formato } \\
\text { de monitoreo }\end{array}$ & $\begin{array}{l}\text { El } 86 \% \text { de la comunidad } \\
\text { fue evaluada. }\end{array}$ \\
\hline
\end{tabular}

Fuente: transferencia Participativa Para la Higiene y el Saneamiento (2012).

En el siguiente cuadro se presentan los indicadores construidos para el seguimiento de la transferencia social de la tecnología (Tabla 5). 
Tabla 5. Indicadores para el seguimiento de la transferencia social de la tecnología.

\section{Indicadores para realizar el seguimiento de la transferencia social de la tecnología}

\begin{tabular}{|c|c|c|}
\hline $\begin{array}{l}\text { Objetivos para medir } \\
\text { la transferencia social }\end{array}$ & Indicador & Criterio \\
\hline \multirow{3}{*}{$\begin{array}{l}\text { Mejorar los niveles } \\
\text { de conocimientos }\end{array}$} & $\begin{array}{l}\text { Presencia de pre-tratamiento } \\
\text { doméstico al sistema de filtración. }\end{array}$ & Cuenta con pre-tratamiento (si/no) \\
\hline & Ubicación adecuada del filtro & Ubicación adecuada (si/no) \\
\hline & Talleres de formación & Asistencia a talleres (No. Personas) \\
\hline \multirow{4}{*}{$\begin{array}{l}\text { Aplicar procesos } \\
\text { de operación } \\
\text { y mantenimiento }\end{array}$} & \multirow{2}{*}{ Uso adecuado del filtro } & En uso (si/no) \\
\hline & & Limpio (si/no) \\
\hline & Herramientas de limpieza del filtro & $\begin{array}{l}\text { Tenencia de herramientas } \\
\text { de limpieza (si/no) }\end{array}$ \\
\hline & $\begin{array}{l}\text { Frecuencia de lavado } \\
\text { y limpieza del filtro }\end{array}$ & Cantidad de veces lavado/semana \\
\hline \multirow{3}{*}{$\begin{array}{l}\text { Mejorar la calidad } \\
\text { de agua }\end{array}$} & Turbidez & NTU \\
\hline & Coliformes fecales & $\mathrm{NMP} / 100 \mathrm{ml}$ \\
\hline & Coliformes totales & $\mathrm{NMP} / 100 \mathrm{ml}$ \\
\hline
\end{tabular}

Fuente: Narváez y Martínez (2017)

Evaluación de eficiencia: respecto a los resultados obtenidos por Laboratorios del Valle, acreditados por la NTC ISO 5667, se determinó la eficiencia de los filtros Sawyer. En el primer muestreo (Tabla 6), el 99\% de los filtros en el parámetro de turbidez está dentro de lo establecido por la normativa colombiana y el $86 \%$ de los filtros está dentro de lo establecido en coliformes totales y fecales. El $14 \%$ de los filtros que no cumplen estos requisitos, fueron utilizados anteriormente en las capacitaciones con la comunidad en temas de uso del filtro, según el CAWST (2011) la inadecuada manipulación de las tecnologías puede repercutir en la eficiencia de remoción de agentes patógenos que pudo ser el caso de dicha eficiencia. 
Tabla 6. Resultados del análisis uno de laboratorio.

\begin{tabular}{|c|c|c|c|}
\hline \multirow[b]{2}{*}{$\mathbf{N}^{\circ}$} & \multicolumn{3}{|c|}{ Muestreo 1} \\
\hline & Turbidez (NTU) & $\begin{array}{l}\text { Coliformes totales } \\
\text { (NMP/100ml) }\end{array}$ & $\begin{array}{l}\text { Escherichia. coli } \\
\text { (NMP/100ml) }\end{array}$ \\
\hline 1 & 0,45 & 0 & 0 \\
\hline 2 & 0,27 & 0 & 0 \\
\hline 3 & 0,27 & 0 & 0 \\
\hline 4 & 0,19 & 0 & 0 \\
\hline 5 & 0,27 & 0 & 0 \\
\hline 6 & 0,24 & 3 & 0 \\
\hline 7 & 0,43 & 20 & 14 \\
\hline 8 & 0,32 & 15 & 4 \\
\hline 9 & 0,35 & 0 & 0 \\
\hline 10 & 0,27 & 0 & 0 \\
\hline 11 & 0,35 & 0 & 0 \\
\hline 12 & 2,66 & 0 & 0 \\
\hline 13 & 0,2 & 0 & 0 \\
\hline
\end{tabular}

Fuente: autor

Por otra parte, el seguimiento realizado al uso, operación y mantenimiento de la tecnología (Figura 2) arrojó que el $100 \%$ de las familias no tienen pre-tratamiento doméstico, el 78,5\% almacena adecuadamente el agua tratada, el 93\% tiene ubicado el sistema en un sitio adecuado, todos los sistemas de tratamiento funcionan correctamente y el $100 \%$ asistió a talleres de formación.

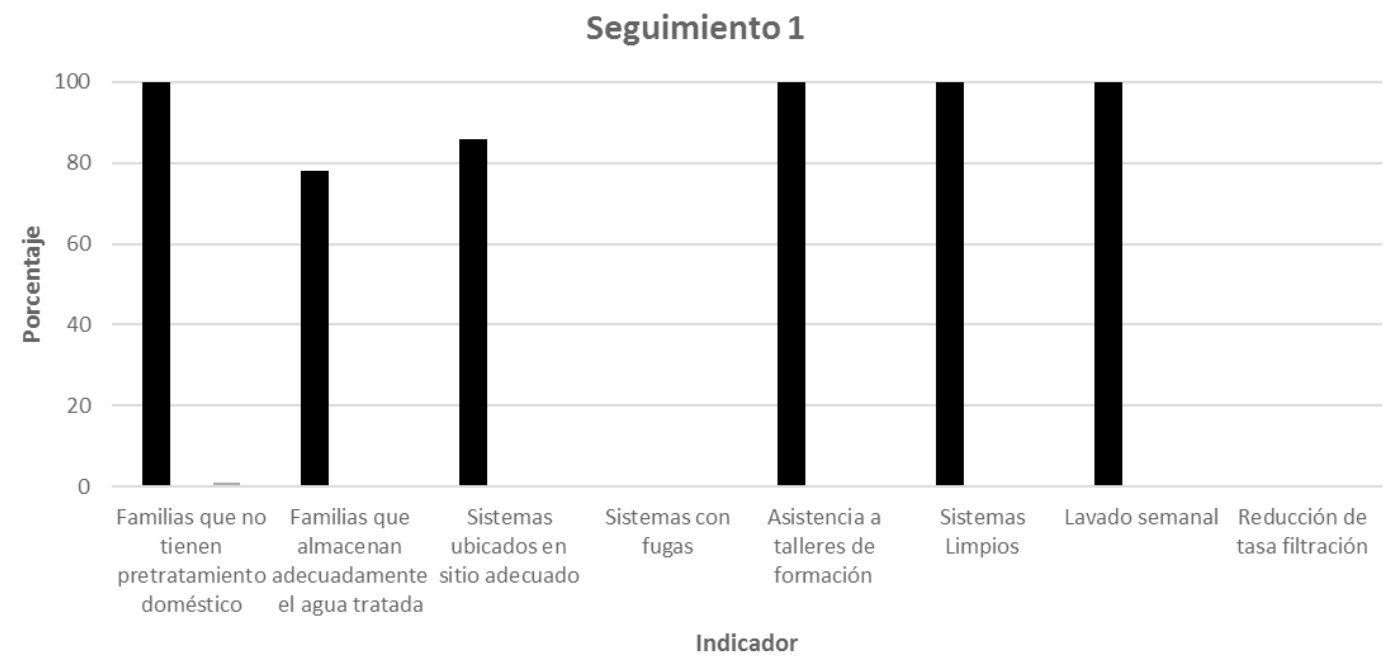

Figura 2. Seguimiento uno de la tecnología.

Fuente: autor. 
En el segundo muestreo (Tabla 7), el 100\% de los filtros en el parámetro de turbidez están dentro de lo establecido por la norma; con respecto a los coliformes totales, el $62 \%$ está dentro de lo estipulado en la norma; y el $77 \%$ cumple con lo estipulado en la norma con respecto a coliformes fecales. Se presenta una disminución de la eficiencia de la tecnología, por lo que fue necesario consolidar una nueva reunión con la comunidad en la que se reforzaron temas del uso y mantenimiento del filtro, además de temas de salubridad e higiene en los hogares.

Tabla 7. Resultados del análisis dos de laboratorio.

\begin{tabular}{|c|c|c|c|}
\hline \multirow[b]{2}{*}{$\mathbf{N}^{\circ}$} & \multicolumn{3}{|c|}{ Muestreo 2} \\
\hline & Turbidez (NTU) & $\begin{array}{l}\text { Coliformes totales } \\
\text { (NMP/100mI) }\end{array}$ & $\begin{array}{l}\text { Escherichia. coli } \\
\text { (NMP/100ml) }\end{array}$ \\
\hline 1 & 0,1 & 0 & 0 \\
\hline 2 & 0,15 & 0 & 0 \\
\hline 3 & 0,09 & 0 & 0 \\
\hline 4 & 0,1 & 0 & 0 \\
\hline 5 & 0,1 & 0 & 0 \\
\hline 6 & 0,43 & 3,6 & 0 \\
\hline 7 & 0,1 & 93 & 3 \\
\hline 8 & 0,2 & 240 & 43 \\
\hline 9 & 0,16 & 0 & 0 \\
\hline 10 & 0,24 & 0 & 0 \\
\hline 11 & 0,22 & 0 & 0 \\
\hline 12 & 0,21 & 210 & 43 \\
\hline 13 & 0,14 & 23 & 0 \\
\hline
\end{tabular}

Fuente: Autor

Como resultado del seguimiento de la tecnología en el segundo muestreo (Figura 3) se evidenció que el $100 \%$ de las familias no tienen pretratamiento doméstico; el 93\% almacena adecuadamente el agua tratada; el 93\% tiene ubicado el sistema en un sitio adecuado; el $7,1 \%$ de los hogares tiene fugas con reducción de tasa de filtración; y el 92,86\% asistió a talleres de formación. 


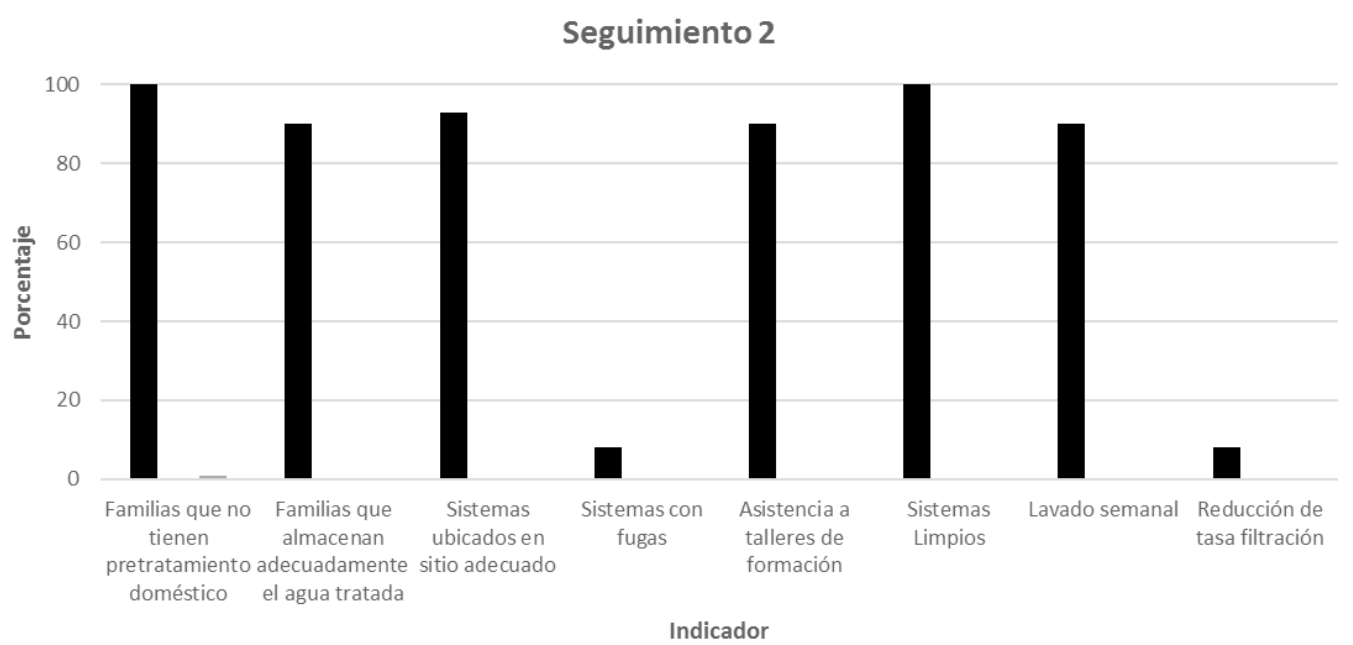

Figura 3. Seguimiento dos de la tecnología. Fuente: autor.

Mediante este seguimiento se determinó un aumento en los coliformes totales y fecales al igual que el porcentaje de la tasa de reducción de filtración, lo que evidencia un inadecuado mantenimiento de la tecnología y genera la disminución de la eficiencia del filtro. Por lo que fue necesario el realizar una capacitación refuerzo en temas de manipulación del filtro.
El tercer y último muestreo (Tabla 8) determinó que el $100 \%$ de los filtros están dentro de lo establecido por la norma en los parámetros de turbidez, coliformes totales y fecales. La OMS (2012) establece que el agua de consumo humano debe estar dentro de lo estipulado por la normativa de cada estado para ser considerada como apta y lograr bienestar en las comunidades.

Tabla 8. Resultados del análisis tres de laboratorio.

\begin{tabular}{|c|c|c|c|}
\hline \multirow[b]{2}{*}{$\mathbf{N}^{\circ}$} & \multicolumn{3}{|c|}{ Muestreo 3} \\
\hline & Turbidez (NTU) & $\begin{array}{c}\text { Coliformes totales } \\
\text { (NMP/100mI) }\end{array}$ & $\begin{array}{l}\text { Escherichia. coli } \\
\text { (NMP/100mI) }\end{array}$ \\
\hline 1 & 0,2 & 0 & 0 \\
\hline 2 & 0,14 & 0 & 0 \\
\hline 3 & 0,08 & 0 & 0 \\
\hline 4 & 0,1 & 0 & 0 \\
\hline 5 & 0,1 & 0 & 0 \\
\hline 6 & 0,22 & 0 & 0 \\
\hline 7 & 0,3 & 0 & 0 \\
\hline 8 & 0,1 & 0 & 0 \\
\hline 9 & 0,1 & 0 & 0 \\
\hline 10 & 0,12 & 0 & 0 \\
\hline 11 & 0,21 & 0 & 0 \\
\hline 12 & 0,1 & 0 & 0 \\
\hline 13 & 0,1 & 0 & 0 \\
\hline
\end{tabular}

Fuente: autor 
Finalmente, el último seguimiento (Figura 4) determinó que el 93\% de las familias almacenó adecuadamente el agua tratada; el $100 \%$ ubicó adecuadamente el sistema; el 93\% asistió al último taller; y el $100 \%$ de los filtros funcionaron de manera adecuada. Todo ello, soportado por las estrategias de uso, manejo y capacitación que de desarrollo paralelamente al proceso de adopción. Con base en esto, se puede afirmar que un proceso planificado entre el facilitador y la comunidad, conllevan a procesos exitosos comunitarios.

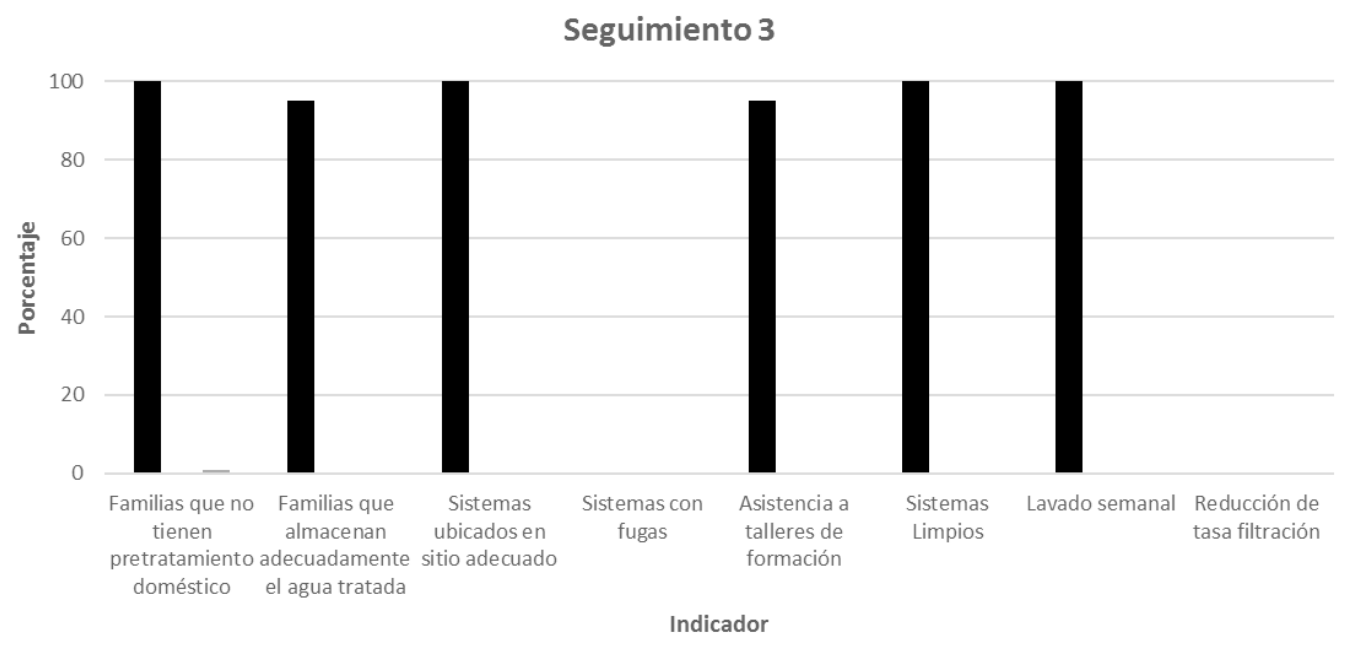

Figura 4. Seguimiento tres de la tecnología.

Fuente: autor

\section{CONCLUSIONES}

La calidad del agua de las dos quebradas que abastecen la planta de tratamiento no presenta ningún tipo de alteración en sus características fisicoquímicas y establece un nivel muy bajo de alteración en sus características microbiológicas; sin embargo, la red de acueducto presenta un nivel alto en la alteración microbiológica y fisicoquímica debido a la colmatación de la tubería que afecta el agua ya tratada.

Las tecnologías de tratamiento no convencional que venían prefabricadas tuvieron mayor aceptación por los expertos que las tecnologías que requerían algún proceso de construcción, argumentando la necesidad de optimizar el tiempo o el riesgo al fracaso por un procedimiento inadecuado que puede ocurrir sin una adecuada orientación; sin embargo, enfatizaron la idea del beneficio de apropiación que tendría el construir el filtro de bioarena con ayuda de la comunidad.
El filtro sawyer tiene una eficiencia del $99 \%$ en la remoción de turbidez, coliformes totales y fecales, si se presenta una adecuada manipulación por parte de las familias, de ahí la importancia de recalcar el constante retrolavado y la higiene a la hora de hacer uso de la tecnología.

El éxito de las tecnologías no convencionales está ligado a la transferencia social que se aplique a lo largo de su implementación, puesto que la apropiación que la comunidad le dé al sistema es vital para su sostenibilidad en el tiempo.

Se recomienda realizar un seguimiento periódico por parte de los líderes comunitarios a lo largo de la vida útil de la tecnología para conocer el comportamiento de la eficiencia en la remoción de agentes patógenos. 


\section{CONTRIBUCIÓN A LA AUTORÍA}

Autor: Álvaro Javier Ceballos Freire, Docente $\mathrm{HC}$ de la Facultad de Ciencias Agrícolas de la Universidad de Nariño, Pasto, Colombia: Formulación y desarrollo de todo el proceso investigativo y de elaboración del artículo científico, análisis de los resultados y acompañamiento en los procesos sociales, con el apoyo de estudiantes de Ingeniería Ambiental de la Universidad de Nariño.

\section{AGRADECIMIENTOS}

Un sincero agradecimiento a la comunidad del corregimiento de San Fernando, municipio de Pasto, Nariño, Colombia, por su responsabilidad al proceso y apoyo. De igual forma, a la Universidad de Nariño a través de la Vicerrectoría de Investigaciones, Postgrados y Relaciones Internacionales (VIPRI), la cual financió el proyecto. A los estudiantes de Ingeniería Ambiental Félix David Rivera Madroñero y Andrés Felipe Zambrano Jácome, por su dedicación y profesionalismo en cada una de las actividades desarrolladas en el proyecto.

\section{LITERATURA CITADA}

Alcaldía municipal de Pasto. (2016-2019). Plan de desarrollo municipal "Pasto educado constructor de paz".

Alleyne, G., y Cohen, D. (2003). Salud, crecimiento económico y reducción de la pobreza. pp. World Health Organization, Geneva. pp. 54-56.

Bejarano, S. y Cisneros, K. (2013). Evaluación financiera de proyectos de cooperación técnica destinados a la generación de ingresos sostenibles. Recuperada de https://repository.cesa.edu. co/bitstream/handle/10726/1193/TMF00304. pdf?sequence $=1$ \&isAllowed $=y$

Carvajal, I. (2012). Mapa de riesgo de la calidad de agua para consumo humano de la vereda La Trinidad y la vereda Tocogua - municipio de Duitama. Boyacá. Recuperada de https://bit. ly/3efyeKUCentre

Centre for affordable water and sanitation technology - CAWST. (2011). Característi-cas filtro Sawyer. Primera edición. Canadá. p. 14.
Centro de Estudios en Planificación, Políticas Públicas e Investigación Ambiental - CEPPIA. (2011). Manual de introducción a la gestión ambiental municipal. Recuperada de https://bit.ly/3edjVX6

Espinosa, A. (2014). Impactos antrópicos en la calidad del agua. Periódico digital de divulgación de la Red del Agua. México: Instituto de Ecología, UNAM. http://www.agua.unam.mx/assets/pdfs/ impluvium/numero03.pdf

Fernández, A. (2012). El agua: un recurso esencial. Química Viva, 11(3), 147-170.

Fondo de las Naciones Unidas para la Infancia UNICEF (2005). El agua potable y el saneamiento básico en los planes de desarrollo. Colombia: Unicef. p. 26.

Fundación por la Social-democracia de las Américas FUSDA. (2008). Medio ambiente y desarrollo: hacia un manejo sustentable del agua. El agua, Recurso Natural y Elemento de Desarrollo. México. p.22.

Grisales, D. (2009). Sistemas no convencionales de tratamiento de agua superficiales para comunidades de desplazados en estado de emergencia (CASO VILLA-CLARÍN). Recuperada de https:// bit.ly/2AOng0q

Instituto Departamental De Salud De Nariño. (2016). Plan de salud territorial- salud para el buen vivir. Diagnóstico de la Situación de Salud. Nariño. Recuperada de https://www.minsalud. gov.co/plandecenal/Paginas/mapa/Analisis-deSituacion-Salud-Narino.pdf

Junta de Acueducto Local. (2017). Actualización del censo sanitario del Corregimiento San Fernando. Nariño. Colombia: Junta de Acueducto Local. p. 10.

Lagares, P \& Puerto, J. (2001). Población y muestra. Técnicas de muestreos. España: Management Mathematics for European Schools, Universidad de Sevilla. pp. 4-11.

León-Agatón, L., Mejía-Gutiérrez, L. F., \& MontesRamírez, L. M. (2015). Caracterización socioeconómica y tecnológica de la producción del plátano en el bajo occidente del departamento de Caldas. Revista Luna Azul, 41, 184-200. http://dx.doi.org/10.17151/luaz.2015.41.11

Leonel H. Narváez N., y Morales D. (2016). Informe de Evaluación de dos sistemas no convencionales para mejorar la calidad de agua de consumo humano en el resguardo indígena Alto Cartagena, adscrito a Camawari, Ricaurte. Colombia: Universidad de Nariño. 
Mazorra J., y González M., (2012). Análisis multicriterio para la selección de la tecnología idónea de tratamiento de aguas residuales en la comunidad de Falía, Delta del Saloum, Senegal. Recuperada de http://www.ocud.es/es/files/ doc720/1javiermazorraaguiar.pdf

Ministerio de Ambiente, Vivienda y Desarrollo Territorial. (2010). Resolución 4716 de 2010. Establecimiento de las condiciones, recursos y obligaciones mínimas que deben cumplir las autoridades sanitarias departamental, distrital y municipal. República de Colombia. Recuperada de https://docs.supersalud.gov.co/ PortalWeb/Juridica/OtraNormativa/R_MPS-MAVDT_4716_2010.pdf

Ministerio de la Protección Social y Ministerio de Ambiente, Vivienda y Desarrollo Territorial. (2007) Resolución 2115. Por medio de la cual se señalan características, instrumentos básicos y frecuencias del sistema de control y vigilancia para la calidad del agua para consumo humano. República de Colombia. Recuperada de https://bit.ly/2Ncjgt2

Ministerio de la Protección Social. (2007). Decreto 1575. Por el cual se establece el Sistema para la Protección y Control de la Calidad del Agua para Consumo Humano. República de Colombia. Recuperada de http://www.minambiente.gov. co/images/GestionIntegraldelRecursoHidrico/ pdf/Disponibilidad-del-recurso-hidrico/Decreto1575-de-2007.pdf

Moreno, J. (2012). Proceso analítico jerárquico (AHP). Fundamentos, metodología y aplicaciones. Recuperada de https://bit.ly/2YbWibO

Murtagh, S. (2009). Enseñanza y aprendizaje en los programas de desarrollo rural. Algunas consideraciones sobre el conocimiento campesino y el rol de los técnicos extensionistas. Recuperada de https://www.aacademica.org/000020/52.pdf

Naciones Unidas Para Los Derechos Humanos NUDH. (2011). El derecho al agua. New York: Oficina del Alto Comisionado de las Naciones Unidas para los Derechos Humanos. p. 64.

Narváez, N., y Martínez, J. (2017). Apropiación social de una tecnología no convencional para el tratamiento del agua en la vivienda, Vereda El Motilón, Pasto, Nariño. Recuperada de http://repositorio.utp.edu.co/dspace/bitstream/handle/11059/7923/628161M385. pdf?sequence $=1$
Instituto Colombiano de Normas Técnicas y Certificación - ICONTEC. (2004). Norma Técnica Colombiana ISO-5667-3. Calidad del agua. Muestreo parte 3: Directrices para la preservación y manejo de muestreas. Recuperada de https:// bit.ly/2UZm80L

Organización de las Naciones Unidas - ONU. (2014). Calidad del agua. Agua fuente de vida. Departamento de Asuntos Económicos y Sociales de Naciones Unidas.

Organización Mundial de la Salud - OMS. (2004). Guías para la calidad del agua potable. Tercera edición. Ginebra, Suiza: Organización Mundial de la Salud. p. 408.

Organización Mundial de la Salud - OMS. (2012). Evaluación de métodos para el tratamiento domésticos de agua: Metas sanitarias y especificaciones de eficiencia microbiológica. Ginebra, Suiza: OMS. p. 78.

Transferencia participativa para la higiene y el saneamiento - PHAST (2012). Guía para la Higiene y el Saneamiento. Organización Mundial de la Salud. (2012). Ginebra.

Patiño, B., Salazar H., y Rodríguez T. (2015). Proyecto Análisis de las implicaciones sociales y económicas de las Autopistas para la Prosperidad en el departamento de Antioquia. Medellín: Gobernación de Antioquia. pp. 72-78.

Alcaldía de Pasto. (2014). Plan de Ordenamiento Territorial de Pasto Territorio Con Sentido. (2014 2027). Clasificación del suelo urbano, rural, suburbano, de expansión y de protección. Colombia.

Pontificio Consejo de Justicia y Paz. (2012). El agua, un elemento esencial para la vida, Adoptar soluciones eficaces. Recuperada de http://troballes.org/wp-content/uploads/Agua-un-elementoesencial-para-la-vida.pdf

Sánchez, D. (2016). Calidad del agua en ríos. Escuela de Ingenieros de Caminos y Puertos de Ciudad Real. Universidad de Castilla.

Subsecretaría de Planeación y Calidad de Pasto. (2018). Índices de morbilidad. Corregimiento de San Fernando. Nariño. Colombia.

Trejos, J. (2007). Selección de beneficiarios para programas sociales. Costa Rica: Instituto de Investigación en Ciencias Económicas. Universidad de Costa Rica. p. 52. 
Conflicto de Intereses Los autores declaran no tener ningún conflicto de intereses

\section{(c) (1) () (2)

Licencia de Creative Commons

Revista de Investigación Agraria y Ambiental is licensed under a Creative Commons Reconocimiento-NoComercial-CompartirIgual 4.0 Internacional License. 
